\title{
Kinetics of domain growth: The relevance of two-step quenches
}

\author{
Chandan Dasgupta \\ School of Physics and Astronomy, University of Minnesota, Minneapolis, Minnesota 55455 \\ Rahul Pandit \\ Department of Physics, Indian Institute of Science, Bangalore 560012, India
}

\begin{abstract}
Domain growth in the wake of a rapid quench from high to low temperatures is studied via Monte Carlo simulations of a ten-state Potts model on a triangular lattice. Quenches in which the temperature is lowered in one step and in two steps are studied. The exponent which characterizes the late-stage growth of domains is found to have different values for one-step and two-step quenches, if standard methods are used to obtain this exponent numerically. However, this difference becomes insignificant if the origin of time for two-step quenches is shifted relative to the origin for one-step quenches to compensate for the larger initial domain sizes in the two-step case. The interpretation of recent experiments on two-step quenches is shown to suffer from a similar ambiguity.
\end{abstract}

\section{INTRODUCTION}

Consider a system which goes from a disordered state to an ordered one as the temperature $\boldsymbol{T}$ is lowered below the ordering temperature $T_{0}$. In addition, let all thermodynamic parameters be such that $q$ ordered phases $(q \geqq 2$ ) coexist for $0 \leqq T<T_{0}$. If such a system is quenched rapidly from a high temperature $\left(T_{h} \gg T_{0}\right)$ to a low temperature $\left(T_{l} \ll T_{0}\right)$, domains of the ordered phases start growing. The kinetics of such domain growth has been studied for many years.' Both the early stages (nucleation and spinodal decomposition) and the late stages of growth have been investigated. We restrict ourselves to the latter.

Over the past few years the study of domain-growth kinetics has been revitalized by extensive numerical simulations of model systems, such as the q-state Potts model $(q \geqq 2)$ in two and three dimensions. ${ }^{2-18}$ One of the principal results obtained from such simulations is that the mean linear domain size

$$
\bar{R}(t) \simeq t^{n(q)}, t \rightarrow \infty,
$$

where the time $t$ is measured from the moment the temperature reaches $T_{l}$ and $n(q)$ is a non-negative exponent that characterizes the late stages of domain growth. If $n(q)=0$, domains might grow logarithmically with increasing $t \overline{(R}(t) \simeq[\ln (t)]^{m(q)}$ as $t \rightarrow \infty$, with $m(q)>0$ ), or as some other complicated sub-power-law function of $t$.

For the case $q=2$ (i.e., the Ising model) with nonconserved order parameter, the nhen $0^{2}$ menological theories of Lifshitz and Allen and Cahn ${ }^{20}$ predict $n(q=2)=\frac{1}{2}$.

This prediction has been confirmed by extensive analytic $^{5,6,2} f_{-25}$ and numerical ${ }^{2,3,16}$ studies. When the order parameter is conserved, the heuristic arguments of Lifshitz and Slyozov ${ }^{26}$ suggest the value $n(q=2)=\frac{1}{3}$, whereas recent renormalization-group calculations by Mazenko et al. ${ }^{25,27}$ predict a logarithmic growth of the average domain size at long times. Lifshitz ${ }^{19}$ and Safran ${ }^{28}$ have argued that, if the order parameter is not conserved and if $q \geqq(d+1)$, where $\mathrm{d}$ is the dimension, then the LifshitzAllen-Cahn ${ }^{19,20}$ mechanism, which yields $n(q=2)=\frac{1}{2}$, becomes inoperative. However, even for $q \geqq(d+1)$, numerical simulations ${ }^{7}$ yield the power law (1), but with $n(q)<\frac{1}{2}$. There have been some attempts ${ }^{29,30}$ to develop phenomenological theories to account for the power law (1) when $q \geqq(d+1)$, but these have not been as successful as their counterparts for $q=\mathbf{2}$.

The growth of domains according to the power law (1) has been observed in experiments. ${ }^{7,20,31,32}$ For large $q(=14)$ the recent experiments of Homma and Clarke ${ }^{33}$ have provided the most direct confirmation of the power law (1). These results agree qualitatively with numerical simulations.?

The exponents that characterize the singularities of thermodynamic functions at critical points are universal, i.e., they do not depend on the details of the microscopic properties of a system, but only on certain relevant parameters. This universality is a result of the divergence of a correlation length. In the late stages $(t \rightarrow \infty)$ of domain growth the length $\bar{R}(t)$ diverges [Eq. (1)], so, by analogy with static critical phenomena, it is not unreasonable to expect that the domain growth exponent $n(q)$ is universal. There is, indeed, some evidence ${ }^{12,15,17,34}$ which indicates that such a universality does exist. However, domaingrowth universality classes seem to be far less universal than their static-critical-phenomena counterparts: A large number of relevant parameters have to be controlled to obtain a given growth exponent. Numerical simulations and phenomenological theories of late-stage growth show that domain-growth universality classes depend on the following: (a) the number of phases $q$ that coexist at low temperatures; 7,12 (b) whether the order parameter is conserved or not conserved; ${ }^{13,15,19-27}$ (c) the type of lattice; ${ }^{35}$ (d) the depth of the quench, ${ }^{36}$ i.e., $T_{0}-T_{l} ;$ (e) whether the domain walls between coexisting phases are sharp or diffuse; ${ }^{11.12}$ ( $f$ ) the presence or absence of impurities; ${ }^{18}(\mathrm{~g})$ the presence or absence of vorticity that gets quenched in; ${ }^{14}$ 
(h) the dimension $d ;^{37}$ and (i) the number of components of the order parameter. ${ }^{38}$

The main purpose of this paper is to show that, if we use standard methods for obtaining the domain-growth exponent $\boldsymbol{n}(q)$ from numerical simulations, then this exponent depends on the mode of quenching: In particular, $n(q)$ depends on whether the system is quenched in one step, $T_{h} \rightarrow T_{l}$, or two, $T_{h-+} T_{0}^{-} \rightarrow T_{l}$. This dependence of $n(q)$ on the mode of quenching has been found experimentally by Homma and Clarke. ${ }^{33}$ It is tempting to conclude, therefore, that the mode of quenching should be added to the list of relevant factors given above. However, we show below that it is possible to argue that the dependence of $n(q)$ on the mode of quenching is not real but arises because of a numerical artifact. The functions $\bar{R}_{1}(t)$ and $\bar{R}_{2}(t)$ (the subscripts 1 and 2 refer to one-step and two-step quenches, respectively) become indistinguishable (given the errors) if the origin of time for twostep quenches is shifted relative to the origin for one-step quenches. This shift compensates for the larger initial domain size in the case of two-step quenches (see below). Experiments in which domain growth can be monitored for long times and long simulations for large systems (far larger than the $200 \times 200$ lattices we have used) should show whether the mode of quenching is truly relevant or irrelevant.

The remainder of this paper is organized as follows. In $\mathrm{Sec}$. II we describe our model, simulations, and results. In Sec. III we reanalyze the experimental data of Homma and Clarke. ${ }^{33}$ We end with some concluding remarks in Sec. IV.

\section{MODEL, SIMULATION, AND RESULTS}

The q-state, ferromagnetic Potts model that we study is defined by the Hamiltonian

$$
H=-J \sum_{\langle i j\rangle} \delta_{S_{i} S_{j}},
$$

where $\mathbf{J}>0$, the variables $S_{i}$ can assume any one of the values $1,2, \ldots, q$ for all sites $\mathrm{i}$ on a two-dimensional, triangular lattice, and $\langle i j\rangle$ are distinct, nearest-neighbor pairs of sites. We use periodic boundary conditions. The model (2) undergoes a first-order phase transition ${ }^{39}$ for $q>4$ at a temperature $T_{0}(q)\left(\simeq 1.095\right.$ in units of $J / k_{B}$ for $q=10$ ). For $T>T_{0}$ the system is disordered: The order parameters

$$
M_{p}=\left\langle q\left(\frac{1}{N} \sum_{i} \delta_{S_{l}, p}\right\rangle-1\right) /(q-1)
$$

are zero for all $p(1 \leqq p \leqq q$, the angular brackets denote a thermal average, and $N$ is the total number of sites). For $T<T_{0}$ there are $q$ coexisting, ordered phases, each one characterized by a positive value of one of the $M_{p}$ 's.

The domain growth kinetics for this model following a one-step quench from infinite temperature has been extensively studied in Ref. 7. Here, we investigate the relevance of two-step quenches by using the standard Metropolis Monte Carlo algorithm ${ }^{40}$ which does not conserve the order parameter. We update the variables $S_{i}$ by stepping sequentially through the lattice. We choose a new, trial value for $S_{i}$ at random. The transition probability for going from the original value of $S_{i}$ to this new trial value is

$$
W=\left\{\begin{array}{l}
\exp \left(-\Delta E / k_{B} T\right), \Delta E>0 \\
1, \Delta E \leqq 0,
\end{array}\right.
$$

where $\mathrm{AE}$ is the change in energy caused by this transition. We measure time in units of Monte Carlo steps per spin $(\mathrm{MCS} / \mathrm{s})$. We begin with a state at infinite temperature $T_{h}$ in which the variables $\left[S_{i}\right]$ assume any one of the values $1,2, \ldots, q$ with equal probability. We then quench the system to a low temperature $T_{l}\left(=T_{0} / 10\right)$ in one of two ways: (1) In the first way we quench directly to $T_{l}$; (2) in the second way we first quench to a temperature slightly below $T_{0}\left(T_{0}^{-}=\mathrm{T}_{0}-0.01\right.$, in units of $\left.J / k_{B}\right)$, anneal the system in this supercooled disordered state for $200 \mathrm{MCS} / \mathrm{s},{ }^{41}$ and then quench a second time to $T_{l}$. We monitor domain growth by calculating the mean size of clusters and the distribution of cluster sizes as a function of time. The origin of time is chosen to be the moment the system is brought to the temperature $T_{l}$. The results we report have been obtained for $q=10$ and for a $200 \times 200$ lattice. We have taken averages over three independent simulations. We have obtained similar results for smaller lattices.

We find that for both one- and two-step quenches $N_{c}(t)$, the total number of clusters at time $t$ is well approximated by the form

$$
N_{c}(t)=A_{T} C /(D+t)^{s},
$$

where $\boldsymbol{A}_{T}$ is the total area and C, $\boldsymbol{D}$, and s are constants. This is one of the standard forms used in analyzing numerical data on domain growth (see Ref. 7, for example). At long times $(t>D)$, the form (4) describes a power-law behavior, and the constant $\boldsymbol{D}$ in the denominator allows for a finite value of $N_{c}$ at $t=0$. By fitting our data for $20 \leqq t \leqq 4000$, we find

$$
s_{1}=0.91 \pm 0.01
$$

and

$$
s_{2}=0.80 \pm 0.01
$$

where the subscripts 1 and 2 refer to one-step and twostep quenches, respectively. These results were obtained by using a nonlinear least-squares-fitting procedure in which all three parameters, $\boldsymbol{C}, \boldsymbol{D}$ and s, were treated as variables to be fitted. The fits are shown in Fig. 1. A fit over the range $100 \leqq t \leqq 4000$ yields similar results: $s_{1}=0.92 \pm 0.01$ and $s_{2}=0.82 \pm 0.02$. Visual inspections of the configurations generated in the simulations indicate that the domains are compact (seeFig. 1 of Ref. 7, for example; quantitative evidence indicating compactness is presented below). For compact clusters, $N_{c}(t) \propto[\bar{R}(t)]^{-2}$, and $n=s / 2$ [cf. Eqs. (1) and (4)]. Our value for $n_{1}$ agrees with the one obtained in Ref. 7.

In the simulation, we also monitor the time dependence of the total excess energy, $\Delta E(t)$, over the ground-state value of $-3 J$ per spin. It is clear from Eq. (2) that $\Delta E(t)$ is approximately proportional to the total boundary length of the domains at time $t$. For compact domains, the total 


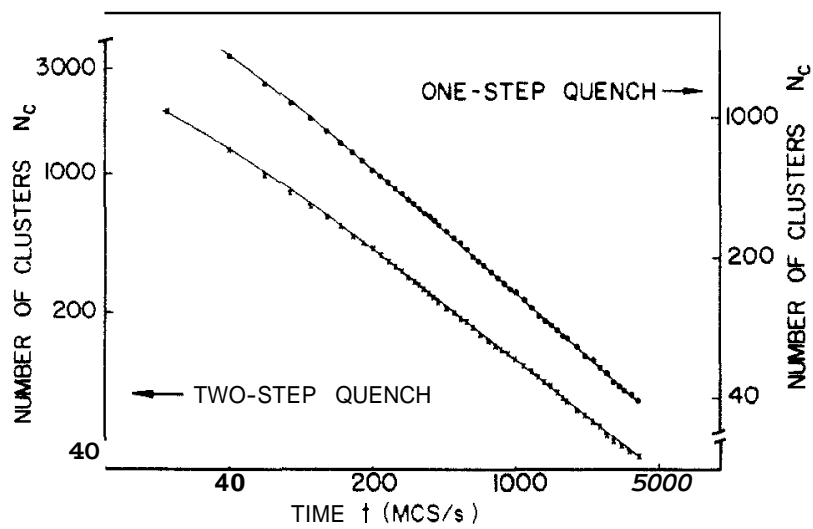

FIG. 1. Variation of the total number of clusters, $N_{c}(t)$, with time $t$ (in units of $\mathrm{MCS} / \mathrm{s}$ ) for one-step (dots) and two-step (crosses)quenches. The solid lines show best fits to the form of Eq. (4). The values of the parameters used in the fits are $C=3.73, D=9.8$, and $s_{1}=0.91$ for the one-step quench, and $C=1.59, \mathrm{D}=9.5$, and $s_{2}=0.805$ for the two-step quench. The total area $\boldsymbol{A}_{T}$ of the $200 \times 200$ triangular lattice used in the simulations is $\sqrt{3} \times 10^{4}$ units.

boundary length should be proportional to the square root of the total number of domains. ${ }^{7,10}$ Thus, at long times, $\Delta E(t)$ is expected to decrease with time as a power law, with an exponent equal to $s / 2$. We find that the variation of $\Delta E(t)$ with time can be fitted quite well by the form (4). The best fits yield the values $0.45 \pm 0.01$ and $0.40 \pm 0.01$ for the exponent associated with $\Delta E(t)$ for one- and two-step quenches, respectively. The fact that these numbers are almost exactly equal to $s / 2$ [cf. Eqs. $(5 a)$ and $(5 b)]$ indicates that the domains are indeed compact, and also provides a consistency check of the calculation.

For both one- and two-step quenches we find that the scaled-cluster-size distribution (see below) assumes a time-invariant form ${ }^{42}$ after $t \simeq 200 \mathrm{MCS} / \mathrm{s}$. This timeinvariant form is practically the same for both quenches. Figure 2 shows the distribution, $P(x)$, of $\mathrm{x} \equiv \log _{10}(A / \bar{A})$, where $A$ is the area of a cluster and $\bar{A}\left(=A_{T} / N_{c}\right)$ is the mean area of clusters for one-step (dashed line) and twostep (solid line) quenches. We have averaged our data over the range $500 \leqq t \leqq 4000$, which corresponds to an average over 95 different configurations. For the one-step quench, the mean of this scaled-cluster-size distribution is -0.23 and its standard deviation is 0.52 . These values are close to the ones quoted for model (2) with $q=12$ in Ref. 7. For the two-step quench the mean of the distribution is -0.245 and the standard deviation is 0.53 . Given the errors of our simulations, the differences between the scaled-cluster-size distributions for one- and two-step quenches are not significant. It is tempting to argue that both the one-step and the two-step scaled-cluster-size distributions are being driven to the same fixed-point distribution, and hence both quenches lie in the same universality class. However, it is not clear to us that two quenches having the same scaled-cluster-size distribution must lie in the same universality class. Other factors such as the shape distribution of the domains and the density of kinks

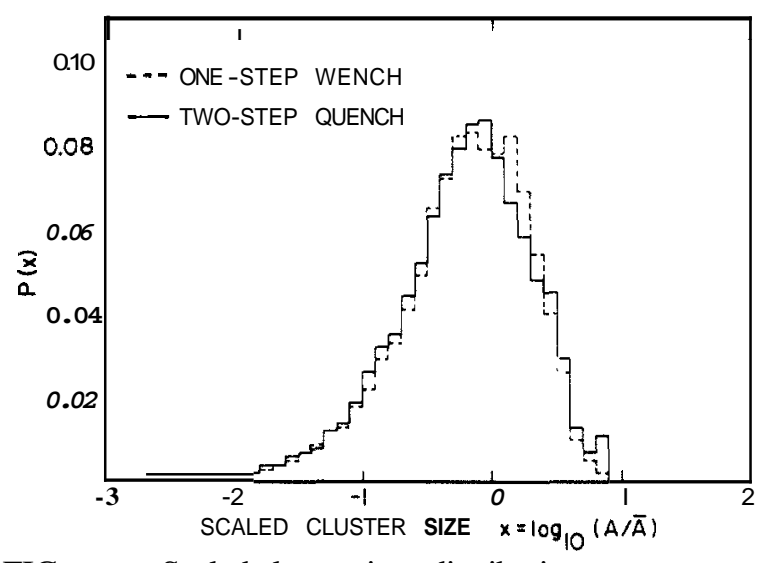

FIG. 2. Scaled-cluster-size distributions, $P(x)$ versus $x \equiv \log _{10}(A / \bar{A})$, where $A$ is the area of a cluster and $\bar{A}$ the mean area of clusters. Dashed and solid lines indicate distributions for one-step and two-step quenches. The data have been averaged over the time range $500 \leqq t \leqq 4000 \mathrm{MCS} / \mathrm{s}$. Given the errors of our simulations, the difference between the two distributions are not significant.

on the domain walls may be important in determining the universality class. In particular, it is known $^{7}$ that for $q \geqq(d+1)$ the growth of domains proceeds via the movement of kinks on the domain boundaries. Thus, if the annealing at $T_{0}^{-}$reduces the average number of kinks per cluster, then the domain-growth exponent $n$ may decrease as a result of this annealing. There is, indeed, some numerical evidence suggesting that such a dependence of the density of kinks on the mode of quenching may be present. We find that the value of the excess energy, $\boldsymbol{A} \boldsymbol{E}$, for every configuration generated after a two-step quench is slightly smaller (by $\simeq \mathbf{2 \%}$ ) than that for a configuration with the same number of clusters generated after a one-step quench. Since $\boldsymbol{A E}$ is a measure of the total boundary length of the domains, this observation suggests that the annealing at $T_{0}^{-}$reduces the average length of the perimeter of a domain. If we consider this result as evidence for a reduction in the average number of kinks per cluster by the annealing process, then we would have a possible explanation of why the observed value of $n_{2}$ is less than that of $n_{1}$. However, the observed differences in the values of $\boldsymbol{A} \boldsymbol{E}$ are within the error bars of our simulation. For this reason, any conclusion based on this observation should be considered tentative, at best. A different, but equally plausible explanation of the observed dependence of $n$ on the mode of quenching is given below.

The annealing of the system at $T_{0}^{-}$yields larger clusters for two-step quenches than for one-step quenches; i.e., at any time $t, N_{c_{2}}(t)<N_{c_{1}}(t)$ and $\bar{R}_{2}(t)>\vec{R}_{1}(t)$. However, we find that plots of $N_{c_{1}}(t)$ and $N_{c_{2}}(t-\Delta t)$ versus $t$ (Fig. 3) for $1000 \leqq t \leqq 4000$ and $\Delta t=140$ are indistinguishable given our error bars $(\simeq 5 \%)$. A shift of the origin of time ${ }^{43}$ compensates for the larger clusters in the case of two-step quenches. It is possible, therefore, that the dependence of the exponent $s$ on the mode of quenching [Eq. (5)] is not real but a mere numerical artifact. If $s$ is determined by fitting $N_{c}(t)$ to the form (4) for $t \gg \Delta t$, 


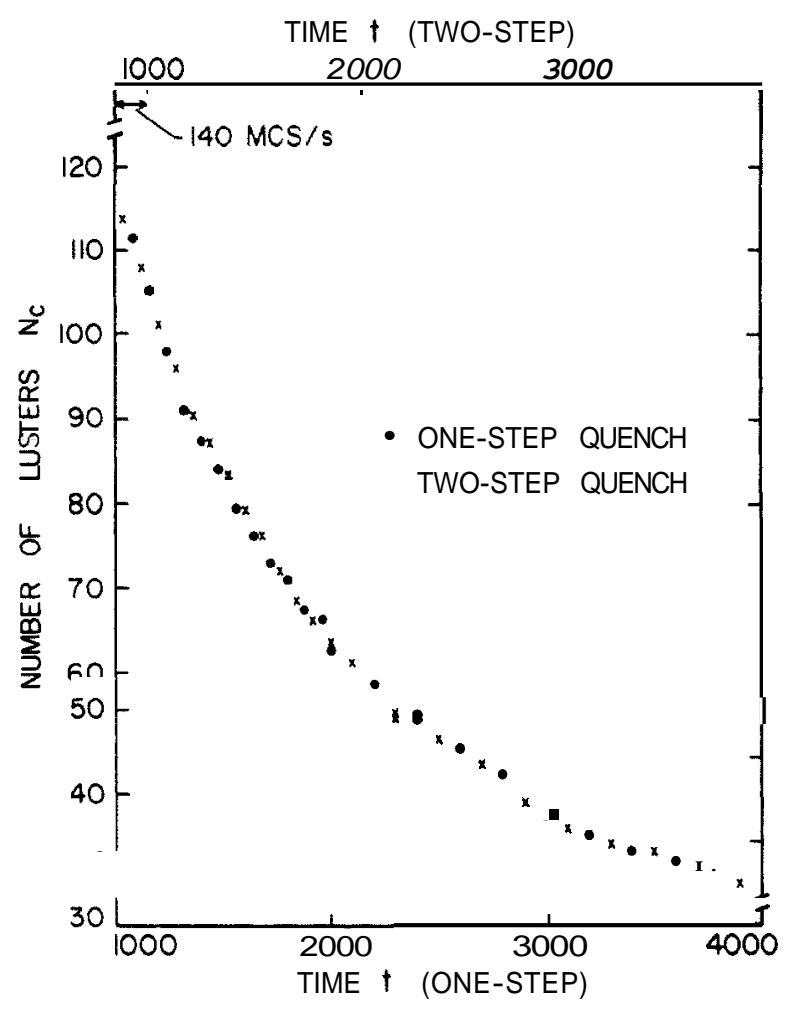

FIG. 3. The total number of clusters $N_{c}(t)$ versus the time $t$ for one-step (dots) and two-step (crosses) quenches. The origin of time for two-step quenches has been shifted by $140 \mathrm{MCS} / \mathrm{s}$ with respect to the origin for one-step quenches (see text). The two plots are indistinguishable (given our errors) over the range $1000 \leqq t \leqq 4000 \mathrm{MCS} / \mathrm{s}$ shown.

then any dependence of $s$ on the mode of quenching must be real. We have tried such fits over the range $1000 \leqq t \leqq 4000$. Unfortunately, our results are not conclusive. By varying the constants $C$ and $D$ in Eq. (4), it is possible to obtain nearly perfect fits to our data for $N_{c}(t)$ $(1000 \leqq t \leqq 4000)$ with any value of $s$ between 0.78 and 0.92. If we fit straight lines to plots of $\ln \left[N_{c}(t)\right]$ versus $\ln (t)$ in the range $1000 \leqq t \leqq 4000$, we obtain $s_{1} \simeq 0.89$ and $s_{2} \simeq 0.83$.

For $t>4000$, the mean area of clusters is a fair fraction ( $\simeq \frac{1}{30}$ ) of the $200 \times 200$ lattices we have used and $N_{c}(t)$ has significant finite-size corrections. Reliable data for $t>4000$ can be obtained only by simulations of model (2) on much larger lattices than the one we have used. Until such calculations are done, it is impossible to decide unambiguously whether $s$ does or does not depend on the mode of quenching.

\section{EXPERIMENTS}

Homma and Clarke ${ }^{33}$ have studied domain-growth kinetics in the wake of rapid quenches from high to low temperatures in graphite intercalated with $\mathrm{SbCl}_{5}$, The intercalant molecules form quasi-two-dimensional superlat- tices which are ordered; 14 ordered phases coexist at room temperature. Homma and Clarke find $\mathbf{n}_{1}=0.52 \pm 0.05$ for a one-step quench and $\boldsymbol{n}_{\mathbf{2}}=0.15 \mathbf{- 0 . 2 5}$ for a two-step quench. $^{33}$ This dependence of $\mathbf{n}(=s / 2)$ on the mode of quenching is in qualitative agreement $\left(n_{2}<n_{1}\right)$ with our results [Eq. (5)] and, like them, is obtained in the standard way [see text after Eq. (4)]. However, we can explain away this dependence of $\mathbf{n}$ on the mode of quenching just as we did for our numerical simulations. Homma and Clarke estimate the mean size of ordered clusters by monitoring the intensity $\mathbf{I}$ of the superlattice peak. A straight-line fit to a plot of $\ln (I)$ versus $\ln (t)$ yields the exponent 2n. Unfortunately, they cannot monitor the growth for very long times because once the clusters are large enough, they get pinned by impurities and the rate of growth decreases dramatically. ${ }^{18,33}$ This pinning occurs after $\simeq 8$ rnin in the case of a one-step quench, but after only $\simeq 5$ min for a two-step quench. This suggests that the cluster configuration that obtains after 8 rnin for a one-step quench is similar to the cluster configuration that obtains after 5 rnin for a two-step quench, i.e., we should choose $A t=3$ rnin (see Fig. 3 and Sec. II). By re. plotting the one-step-quench data of Homma and Clarke, we find that a shift of the origin of time by 3 rnin lowers the value of the effective exponent to 0.2 , which falls right in the middle of Homma and Clarke's estimate of $n_{2}=\mathbf{0 . 1 5}-\mathbf{0 . 2 5}$. Thus the apparent discrepancy between $n_{1}$ and $n_{2}$ can be explained away by such a shift of the origin of time.

\section{CONCLUDING REMARKS}

We have shown that domain growth needs to be monitored for very long times before we can unambiguously decide whether the domain-growth exponent $n$ does or does not depend on the mode of quenching. Such longtime data are not yet available, neither from numerical simulations (Sec. II) nor from experiments (Sec, III), Numerical simulations of model (2) on large lattices $(\simeq 400 \times 400)$ and experiments on samples with a low concentration of impurities must be carried out to obtain reliable long-time data.

Real-space renormalization groups $^{25,27}$ and Monte Car. lo renormalization groups ${ }^{44}$ have been used recently to study late-stage domain growth in the two-dimensional Ising model. If such renormalization groups can be developed for the study of domain growth in model (2) for $q>\mathbf{2}$, then they can be used to examine the relevance of the mode of quenching. ${ }^{45}$

We close with a word of caution. The size of the lattice and the length of the MC runs used in the present work are comparable to those used in nearly all of the existing numerical studies ${ }^{2-18}$ of domain-growth kinetics. The method of analysis used by us is also very similar to that employed in previous studies. These facts suggest that some of the existing numerical results about the kinetics of domain growth may also involve ambiguities similar to those encountered in the present work. In particular, our 
experience with this simulation leads us to believe that values of the exponent $n$ obtained by fitting MC data for a few thousand times steps to a form like Eq. (4) or to a power-law form like Eq. (1) may not be accurate. Thus, any conclusion about the universality classes of late-stage domain growth based solely on small differences in numerically determined values of the growth exponent $n$ may not be reliable.

\section{ACKNOWLEDGMENTS}

We would like to thank G. S. Grest, T. V. Ramakrishnan, O. T. Valls, and F. C. Zhang for helpful discussions. This study was initiated while one of us (C. D.) was visiting the Department of Physics, Indian Institute of Science, Bangalore. This work was supported in part by the University Grants Commissions, India. C. D. acknowledges support from the Alfred P. Sloan Foundation.

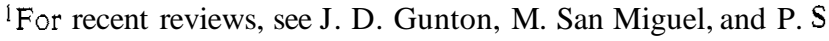
Sahni, in Phase Transitions and Critical Phenomena, edited by C. Domb and J. L. Lebowitz (Academic, New York, 1983), Vol. 8, pp. 267-466; J. D. Gunton and M. Droz, Introduction to the Theory of Metastable and Unstable States (SpringerVerlag, Heidelberg, 1983).

${ }^{2}$ M. K. Phani, J. L. Lebowitz, M. H. Kalos, and O. Penrose, Phys. Rev. Lett. 45, 366 (1980).

${ }^{3}$ P. S. Sahni, G. Dee, J. D. Gunton, M. K. Phani, J. L. Lebowitz, and M. Kalos, Phys. Rev. B 24, 410 (1981).

${ }^{4}$ P. S. Sahni and J. D. Gunton, Phys. Rev. Lett. 45, 369 (1980); 47, 1754(1981).

${ }^{5}$ P. S. Sahni, G. S. Grest, and S. A. Safran, Phys. Rev. Lett. 50 , 60 (1983).

6S. A. Safran, P. S. Sahni, and G. S. Grest, Phys. Rev. B 28, 2693 (1983).

7P. S. Sahni, G. S. Grest, M. P. Anderson, and D. J. Srolovitz, Phys. Rev. Lett. 50, 263 (1983);P. S. Sahni, D. J. Srolovitz, G. S. Grest, M. P. Anderson, and S. A. Safran, Phys. Rev. B 28, 2705 (1983)

${ }^{8}$ K. Kaski, M. C. Yalabik, J. D. Gunton, and P. S. Sahni, Phys. Rev. B 28, 5263 (1983).

${ }^{9}$ A. Sadiq and K. Binder, Phys. Rev. Lett. 51, 674 (1983).

${ }^{10}$ A. Sadiq and K. Binder, Surf. Sci. 128, 350 (1983).

${ }^{11}$ O. G. Mouritsen, Phys. Rev. B 28, 3150 (1983); Phys. Rev. Lett. 56, 850 (1986).

${ }^{12}$ G. S. Grest, D. J. Srolovitz, and M. P. Anderson, Phys. Rev. Lett. 52, 1321 (1984).

${ }^{13}$ G. S. Grest and P. S. Sahni, Phys. Rev. B 30, 226 (1984).

${ }^{14}$ G. S. Grest and D. J. Srolovitz, Phys. Rev. B 30, 6535 (1984).

${ }^{15}$ A. Sadiq and K. Binder, J. Stat. Phys. 35, 517 (1984).

${ }^{16}$ E. T. Gawlinski, M. Grant, J. D. Gunton, and K. Kaski, Phys. Rev. B 31, 281 (1985).

$17 \mathrm{~J}$. Viñals and J. D. Gunton (unpublished).

${ }^{18}$ G. S. Grest and D. J. Srolovitz, Phys. Rev. B 32, 3014 (1985); D. J. Srolovitz and G. S. Grest, ibid. 32, 3021 (1985); D. A. Huse and C. A. Henley, Phys. Rev. Lett. 54, 2708 (1985).

${ }^{19}$ I. M. Lifshitz, Zh. Eksp. Teor. Fiz. 42, 1354 (1962) [Sov. Phys.-JETP 15,939(1962)].

${ }^{20}$ S. M. Allen and J. W. Cahn, Acta Metall. 27, 1085 (1979).

${ }^{21}$ K. Kawasaki, M. C. Yalabik, and J. D. Gunton, Phys. Rev. A 17, 455 (1978).

${ }^{22}$ K. Kawasaki and T. Ohta, Prog. Theor. Phys. 67, 147 (1982); $68,129(1982)$

${ }^{23}$ T. Ohta, D. Jasnow, and K. Kawasaki, Phys. Rev. Lett. 49, 1223 (1982).

${ }^{24}$ M. Grant and J. D. Gunton, Phys. Rev. B 28, 5496 (1983).

${ }^{25}$ G. F. Mazenko and O. T. Valls, Phys. Rev. B 30, 6732 (1984); G. F. Mazenko, O. T. Valls, and F. C. Zhang, ibid. 31, 4453 (1985).

${ }^{26}$ I. M. Lifshitz and V. V. Slyozov, J. Phys. Chem. Solids 19, 35 (1961).
${ }^{27}$ G. F. Mazenko, O. T. Valls, and F. C. Zhang, Phys. Rev. B 32, 5807 (1985); G. F. Mazenko and O. T. Valls, ibid. 33, 1823 (1986).

${ }^{28}$ S. A. Safran, Phys. Rev. Lett. 46, 1581 (1981).

${ }^{29}$ H. Furukawa, Phys. Rev. A 29, 2160 (1984).

${ }^{30} \mathrm{~K}$. Kawasaki (unpublished).

${ }^{31}$ T. Hashimoto, K. Nishimura, and Y. Takeuchi, Phys. Lett. 65A, 250 (1978).

${ }^{32} \mathrm{G}$. -C. Wang and T.-M. Lu, Phys. Rev. Lett. 50, 2014 (1983).

${ }^{33}$ H. Homma and R. Clarke, Phys. Rev. Lett. 52, 629 (1984).

${ }^{34}$ F. C. Zhang, O. T. Valls, and G. F. Mazenko, Phys. Rev. B 31, 1579(1985).

${ }^{35}$ For Potts models with $q>2$ on a square lattice, quenches to very low temperatures $\left(T_{l} \ll T_{0}\right)$ yield frozen configurations which evolve very slowly in time; however, the power law (1) is obtained for all $T_{l}$ on a triangular lattice (Ref. 7).

${ }^{36}$ The slow evolution of quenched configurations on a square lattice (Ref. 35) crosses over to the power law (1) obtained on a triangular lattice for $0.5 T_{0} \leqq T_{l}$ (Ref. 7).

${ }^{37}$ For $q=2$ and the case of a nonconserved order parameter, $n(q=2)$ does not depend on the dimension $d$ (Refs. 2, 3, 19, 20, and 23); however, the dynamic structure factor assumes a scaling form with a dimension-dependent scaling function (Ref. 23). The dependence of $\boldsymbol{n}(\boldsymbol{q}>2)$ on $\mathrm{d}$ is not known.

${ }^{38} \mathrm{G}$. F. Mazenko and M. Zanetti [Phys. Rev. Lett. 53, 2106 (1984)] have studied the growth of order in a system in which the order parameter has an infinite number of components. For the case of a nonconserved order parameter they find $n=\frac{1}{2}$ in agreement with the Lifshitz-Allen-Cahn results (Refs. 19 and 20) for a one-component order parameter; however, when the order parameter is conserved, they find $n=\dagger$ in contrast to both the Lifshitz-Slyozov result (Ref. 26), and the renormalization-group result (Ref. 27) for a onecomponent order parameter.

${ }^{39}$ For a review of the equilibrium properties of Potts models, see F. Y. Wu, Rev. Mod.Phys. 54, 235 (1982).

${ }^{40} \mathrm{~K}$. Binder, in Monte Carlo Methods in Statistical Physics, edited by K. Binder (Springer, Berlin, 1979), pp. 1-45.

${ }^{41}$ The domain-growth exponent is expected to depend on the time for which the system is annealed at $T_{0}^{-}$. We find, however, that the configurations at $T_{0}^{-}$after $t=100 \mathrm{MCS} / \mathrm{s}$ and $\mathrm{t}=200 \mathrm{MCS} / \mathrm{s}$ are similar. Thus, if the system is annealed at $T_{0}^{-}$for a time longer than $\simeq 100 \mathrm{MCS} / \mathrm{s}$, but shorter than the nucleation time of the ordered phase, the domain-growth exponent should be independent of the annealing time. We have not investigated the dependence of the domain-growth exponent on the annealing time for times less than $100 \mathrm{MCS} / \mathrm{s}$.

${ }^{42}$ The temporal self-similarity of the scaled-cluster-size distribution has been demonstrated explicitly in Ref. 7 for a one-step quench of the $q=12$ model. Our results for both one- and two-step quenches with $q=10$ are qualitatively very similar.

${ }^{43} \mathrm{We}$ obtain $\mathrm{At}=140 \mathrm{MCS} / \mathrm{s}$ as follows: we evaluate 
$\sum_{t=1000}^{4000}\left\{\left[N_{c_{1}}(t)-N_{c_{2}}(t-\Delta t)\right]^{2} /\left[N_{c_{1}}(t)\right]^{2}\right\}$ for various values of $\Delta t$ and then find the value of $\Delta t(=140 \mathrm{MCS} / \mathrm{s})$ which minimizes this expression.

44J. Viñals, M. Grant, M. San Miguel, J. D. Gunton, and E. T.
Gawlinski, Phys. Rev. Lett. 54, 1264(1985).

${ }^{45}$ It is unlikely that the Lifshitz-Allen-Cahn mechanism (Refs. 19 and 20) for $q=2$ will be affected by the mode of quenching. 